\title{
Mood disorder in the perinatal period
}

\author{
A woman with bipolar disorder who became pregnant during a long period of severe depression \\ describes how the support of a perinatal team was vital to her postnatal recovery
}

\author{
patient ${ }^{1}$, Nisha Shah consultant perinatal psychiatrist ${ }^{2}$ \\ ${ }^{1}$ London; ${ }^{2}$ Camden and Islington NHS FoundationTrust, Highgate Mental Health Centre, London N19 5NX, UK
}

This is one of a series of occasional articles by patients about their experiences that offer lessons to doctors. The BMJ welcomes contributions to the series. Please contact Peter Lapsley

(plapsley@bmj.com) for guidance.

With a history of depression since my teens, I was finally diagnosed with bipolar disorder in my early 30 s, when I was hospitalised with a manic episode in 2008. I was psychotic and dangerously out of control. Shortly after discharge I went into a depression that effectively paralysed me for the next 18 months. I was a shadow of myself, existing (in relentless pain) rather than living. I think you can only use metaphors to try and describe what severe depression feels like-words simply do not convey how bad it is. It was during this episode of depression that I became pregnant. I was shocked and scared when I found out-how on earth would I be able to take care of a baby when I could barely even get out of bed? I remember telling my community psychiatric nurse that I planned to commit suicide straight after the baby was born but that I would try to hold on until then.

At the time, I was taking lithium, which carries a raised risk of fetal heart malformations. By the time I discovered I was pregnant I was told it was too late to come off it, and given that I was seriously suicidal, my psychiatrist recommended I continue taking it. Waiting for the heart scan at 20 weeks was an extremely anxious time. I was referred to the new perinatal mental health service at the Whittington Hospital, London. I was thoroughly assessed by a perinatal psychiatrist at 16 weeks. She told me that I had a 1 in 2 chance of a relapse after the birth. It was around this time that my depression finally began to lift, so learning that the odds of my becoming ill again were so high was very upsetting.

\section{Prebirth planning}

The perinatal team organised a multidisciplinary prebirth planning meeting when I was 32 weeks along, which I, my husband, and all the relevant health professionals involved in my care attended. This included my community mental health team, my general practitioner, and my named midwife. It was reassuring to see all these people in the same room. One of my main concerns was whether to discontinue the lithium so that I could breastfeed. It was very useful to hear the viewpoints of several different professionals, including a consultant paediatrician.

The outcome of the meeting was a birth plan, which contained a sort of contingency plan for the eventuality that I fall ill, along with a list of warning signs that I had identified with the help of my husband. The birth plan proved vital to my recovery after the birth, and I found that my early recognition of the warning signs was half the battle.

\section{The birth}

I had planned on having a completely natural birth. Instead, I was induced for almost 30 hours and ended up having an emergency caesarean section. My baby then aspirated meconium and had to be resuscitated. This was very traumatic. The first feeling I had after my baby was born was that I wanted to die because I was convinced that my baby would die. I became hysterical and then went into a state of shock and vomited. My baby was taken to the neonatal intensive care unit before I'd had a chance to meet her, and after that I felt a guilty reluctance to go and see her. This indifference carried on for the next few days as I became completely preoccupied with myself.

I couldn't sleep despite feeling exhausted, having not really slept for two nights, and I had to be given sleeping pills. My mind was racing, and even relaxing music would stimulate me. Fortunately I had my own room, which meant I was shielded to some extent from the noisy, stressful ward. I remember thinking that everything seemed sharper and brighter and that the ward notice board suddenly seemed blindingly red. I became obsessed with the idea of going on daytime television to tell my birth story. I was escalating into mania, and yet some of the midwives on the ward did not seem to appreciate how scary this was for me. I remember one of them saying to me when I told 
her I thought I was becoming ill, "You don't seem unwell.” I replied with something like, "By the time it's obvious to you it's too late for me." I was very agitated and recall texting my sister to say that you have to be acting like a crazy person or killing yourself for people to take you seriously.

\section{The aftercare}

My postnatal care involved many different healthcare professionals. This was exhausting and stressful, and having to be seen by new people every day was at times detrimental to my mental health. The two constants were my named midwife, whom I'd seen regularly throughout my pregnancy, and the perinatal team, who came to see me almost every day after the birth during my two week hospital stay. The perinatal team closely monitored my lithium levels and made sure that the midwives on the ward chased up and administered the appropriate medication.

The weekend after the birth, when I was at my most vulnerable, unfortunately coincided with the perinatal team being off duty. Weekend cover was provided by the psychiatric liaison team, who were unfamiliar with my history. I remember recounting my birth story to the two liaison team nurses, telling them it was the most traumatic thing that had ever happened to me. Then, before I had finished, one of the nurses' beepers went off and she left the room without saying anything to me. Shortly afterwards, the other nurse walked out too, again with no explanation. I waited for probably over half an hour, becoming more and more anxious, to the point where I had palpitations and was actually shaking. I eventually stormed out of the room declaring I was going to see my baby and it was only then that they paid attention. The visit from the liaison team did nothing more than aggravate my already heightened mental state. What followed was a series of mental health workers, recruited by the liaison team, who were meant to provide me with one to one support. However, as they didn't seem to be properly trained it was counterproductive. I remember becoming very distressed when one of them came into my room when I was having private time with my family and talked at great length about himself and his career.
Ultimately, what helped me was having continuity of care and a team of specialists who had knowledge of my history and who were able to assess my mental health in the context of my birth. Without the high level of care and intervention that I received during my pregnancy and after the birth, I am certain that my chances of a relapse would have been much higher.

\section{Looking ahead}

My baby is now a happy, healthy 1 year old. The first few months were stressful, particularly as my moods were so volatile, but with the help of a very supportive husband and family and continuing treatment from my community mental health team (which included valuable psychological therapy) I managed to narrowly escape an episode of mental illness, and I have remained well ever since. I count myself very lucky. Not all women have the supportive family that I have, and most women who become ill during pregnancy or after the birth do not have ongoing mental health support. This problem needs to be resolved.

My husband and I are now thinking about the possibility of a second child. Although I am still taking lithium, my situation is very different from that of two years ago. I am well and now have the opportunity to plan for the next pregnancy. When the time comes, I hope that I can access the perinatal mental health service once again.

Competing interests: Both authors declare: no support from any organisation for the submitted work; no financial relationships with any organisations that might have an interest in the submitted work in the previous three years; no other relationships or activities that could appear to have influenced the submitted work.

Provenance and peer review: Not commissioned; not externally peer reviewed.

Accepted: 25 November 2011

Cite this as: BMJ 2012;344:e1209

๑ B BMJ Publishing Group Ltd 2012 


\section{What made a difference to me?}

- Having a specialist perinatal mental health team

- Having continuity of care. For example, being seen by the same midwife and obstetrician throughout my pregnancy and after the birth was extremely important

- Good communication between professionals and agencies. Good communication was crucial to my care, especially after discharge. Unfortunately, the lines of communication between the hospital, my community mental health team, the liaison team, social services, and even within teams themselves broke down more often than not, and frequently I ended up being the one passing on information. This is surely taking patient led care a step too far

- Having compassionate midwives on the ward who had an understanding of mental health

- Having a private room postnatally

\section{My overall thoughts}

- Perinatal mental health teams provide an invaluable service to women at what can be the most vulnerable time of their life

- The role of these teams in recognising those women who are potentially at risk, and supporting them during both their antenatal and postnatal period, is critical in the prevention of mental illness. This is all the more reason to further resource and expand this Cinderella service

- It would be beneficial for midwives to gain some training in mental health

\section{A clinician's perspective}

Specialist mental health services have grown in a somewhat piecemeal fashion, influenced by geography, politics, and personality. Although the French psychiatrist Jean-Étienne Dominique Esquirol described postnatal depression as early as 1838 , the Section of Perinatal Psychiatry at the Royal College of Psychiatrists was inaugurated only in 1996. In 2004 the deaths of Daksha Emson, a consultant psychiatrist, and of her baby daughter provided impetus for further service development.

This patient came to our newly established perinatal psychiatric service at the Whittington Hospital in 2010. She had not benefited from preconception counselling as advocated by the guidelines of the National Institute for Health and Clinical Excellence and was taking lithium as prophylaxis against relapse of her bipolar affective disorder. Close working with our maternity colleagues ensured appropriate monitoring of the cardiac development during pregnancy. Lithium is associated with several cardiac anomalies, including Ebstein's, with a risk of cardiac defects that is 7.7 times higher than that of the general population.

Dose titration of lithium during pregnancy necessitates close monitoring to compensate for increased blood volume in the second trimester and to detect any other serious disorder-for example, pregnancy induced hypertension or diabetes, suggested by a reduction in glomerular filtration rate. Breastfeeding is contraindicated with lithium.

Medication management is only a small part of the functioning of a perinatal psychiatry service. Liaison with many other professionals from both health and social services is imperative. This is ideally documented at the 32 week multidisciplinary prebirth planning meeting mentioned by our patient in her article.

Risk assessment and management must incorporate mother and baby and the relationship between them. There are other important differences from other psychiatric specialities: puerperal psychotic illnesses have distinctive epidemiology, with the risk of postnatal relapse of bipolar illness being $40-70 \%$. The onset of such an illness is often sudden and the presentation more "organic," with affective lability and visual hallucinations being common.

Perinatal services also have responsibilities to train staff working in acute care. Busy midwives must understand the role of sleep deprivation in triggering relapse, so that women at risk can be placed in side rooms if safe, or provide nursing care to minimise other relapse triggers. We work collaboratively with our patients, explaining risks of untreated illness and balancing these against iatrogenic risks. Evidence, though accumulating, cannot be acquired through randomised control trials.

Controversy still exists regarding the ideal configuration of services. Equity of access in England varies as the 110 beds in mother and baby units are distributed unevenly across the country (number supplied by Professor L Howard, personal communication). Many services have grown from existing liaison services, which in London, for example, allow women living in one borough to receive care in another, but which cannot provide a community based service. Our patient lives outside the borough that has commissioned the Whittington service so should have received only a single assessment by us before return of her care to her locality mental health team. Without specialist input the risk of relapse could have been higher, with potential consequences for her, her baby, and her family and social contacts. And, as so often, prevention is better than cure.

Nisha Shah, consultant perinatal psychiatrist 